\title{
Silicon forms a rich diversity of aliphatic polyol complexes in aqueous solution.
}

\author{
Bradley M. Vis, Jiali Wen,† Soren K. Mellerup,‡ Roger D. Merchant, Robert C. Mawhinney, \\ Stephen D. Kinrade* \\ Department of Chemistry, Lakehead University, 955 Oliver Road, Thunder Bay, ON P7B 5E1, \\ Canada
}

\section{Supporting Information}

Table S1. Gas phase B3LYP/6-311++G(d,p) predicted relative stabilities $\left(\Delta E_{\text {elec }}\right)$, equilibrium population $(P)$, and $\mathrm{O}-\mathrm{C}-\mathrm{C}-\mathrm{O}$ dihedral angles of the most stable threitol and erythritol conformers as per Jesus et al. ${ }^{1}$

\begin{tabular}{cccccc}
\hline Conformer & $\Delta E_{\text {elec }}{ }^{a}$ & $P / \%$ & \multicolumn{2}{c}{ Dihedral angles ${ }^{b} / \mathrm{deg}$} \\
& $/$ kcal mol $^{-1}$ & & $\phi_{2}$ & $\phi_{3}$ & $\phi_{4}$ \\
\hline ER1-3 & 3.07 & 18.16 & 168.6 & 58.3 & 52.6 \\
ER4-5 & 4.01 & 19.37 & 53.0 & 55.0 & 52.8 \\
ER6 & 4.56 & 17.53 & 62.1 & -57.5 & -53.1 \\
ER7-8 & 4.43 & 12.16 & -49.3 & 81.6 & -55.2 \\
ER9 & 4.72 & 12.59 & -72.9 & 160.9 & 178.0 \\
ER10 & 4.94 & 20.18 & 56.7 & -168.5 & 60.3 \\
TR1 & 0.00 & 39.08 & 49.1 & -72.9 & 49.1 \\
TR2 & 0.19 & 40.62 & 58.6 & -62.5 & 58.6 \\
TR3 & 2.58 & 20.30 & -55.2 & -73.8 & 48.8 \\
TR4 & 2.97 & 0.00 & 54.7 & 174.2 & 46.8 \\
\hline
\end{tabular}

${ }^{a}$ Electronic energies relative to TR1 (D-threitol; $E_{\text {elec }}=-459.363609$ Hartree). ${ }^{b} \phi_{2}=\mathrm{O} 1-\mathrm{C} 1-\mathrm{C} 2-$ $\mathrm{O} 2 . \phi_{3}=\mathrm{O} 2-\mathrm{C} 2-\mathrm{C} 3-\mathrm{O} 3 . \phi_{4}=\mathrm{O} 3-\mathrm{C} 3-\mathrm{C} 4-\mathrm{O} 4$. 
a)

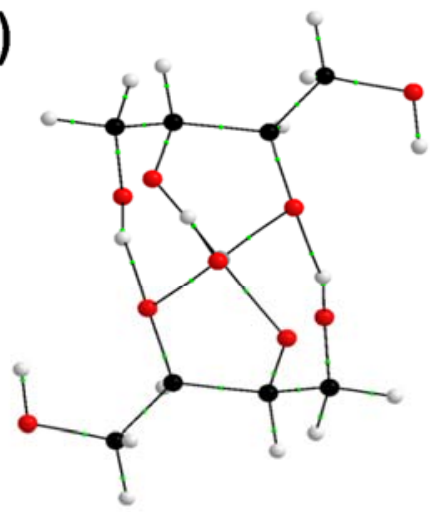

$$
\begin{gathered}
\underline{p}^{0}(2 a, 3 e)(2 a, 3 e) \\
0 \mathrm{kcal} \mathrm{mol}^{-1}
\end{gathered}
$$

b)

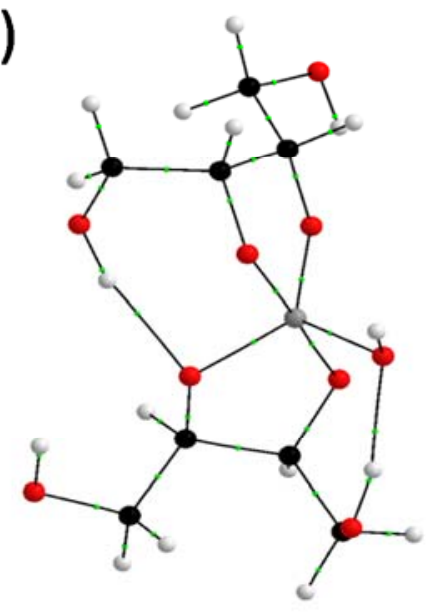

$\underline{\mathrm{p}}^{0}(2 \mathrm{a}, 3 \mathrm{e})(2 \mathrm{e}, 3 \mathrm{a})$

$0 \mathrm{kcal} \mathrm{mol}^{-1}$

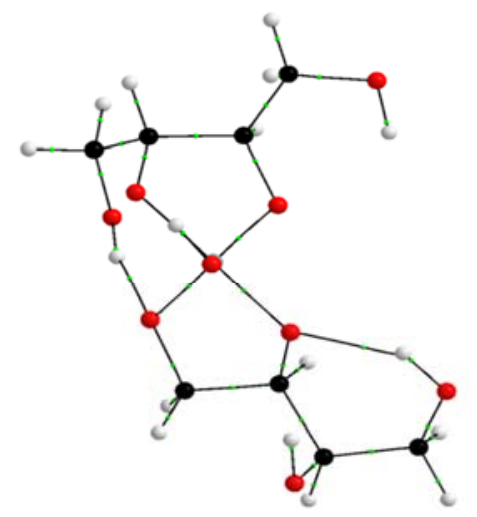

$\underline{P}^{0}(1 e, 2 a)(2 a, 3 e)$

$1.71 \mathrm{kcal} \mathrm{mol}^{-1}$

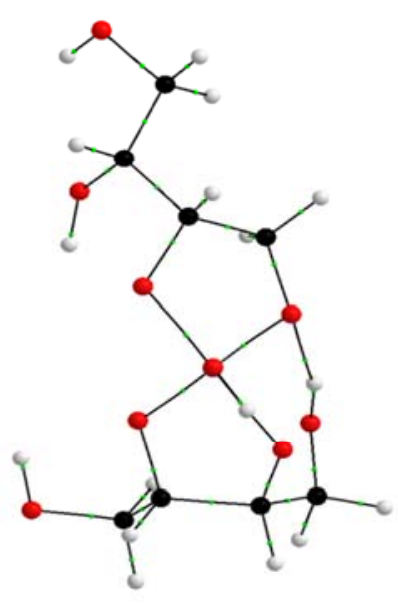

$\underline{p}^{0}(2 a, 3 e)(3 a, 4 e)$

$0.06 \mathrm{kcal} \mathrm{mol}^{-1}$

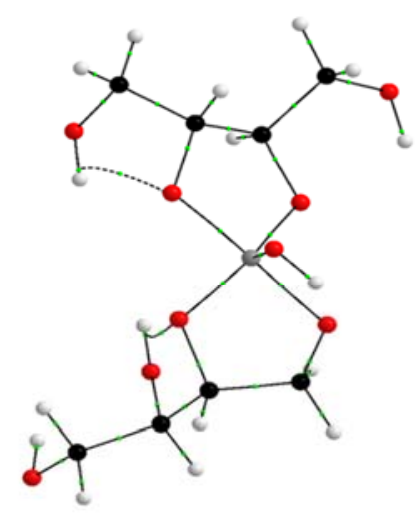

$\underline{p}^{0}(1 a, 2 e)(2 a, 3 e)$

$2.45 \mathrm{kcal} \mathrm{mol}^{-1}$

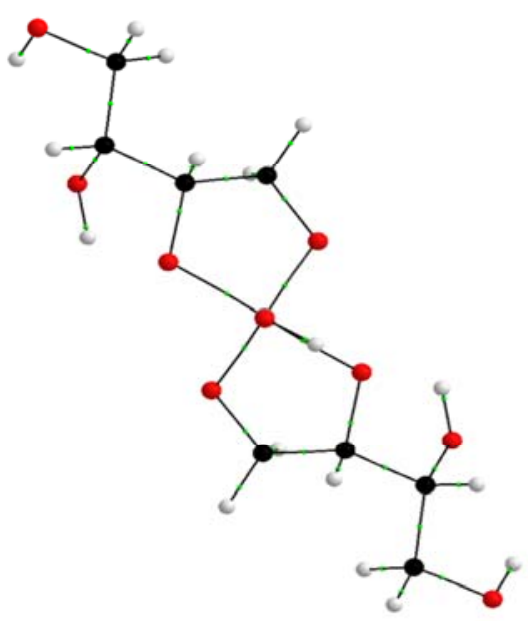

$\underline{p}^{0}(3 a, 4 e)(3 a, 4 e)$

$0.56 \mathrm{kcal} \mathrm{mol}^{-1}$

Figure S1. The three most abundant $\underline{\mathrm{P}}^{0}(=\mathrm{L})_{2}$ complexes predicted for a) threitol and $\mathrm{b}$ ) erythritol using B3LYP/6-31++G(d,p) IEFPCM. 


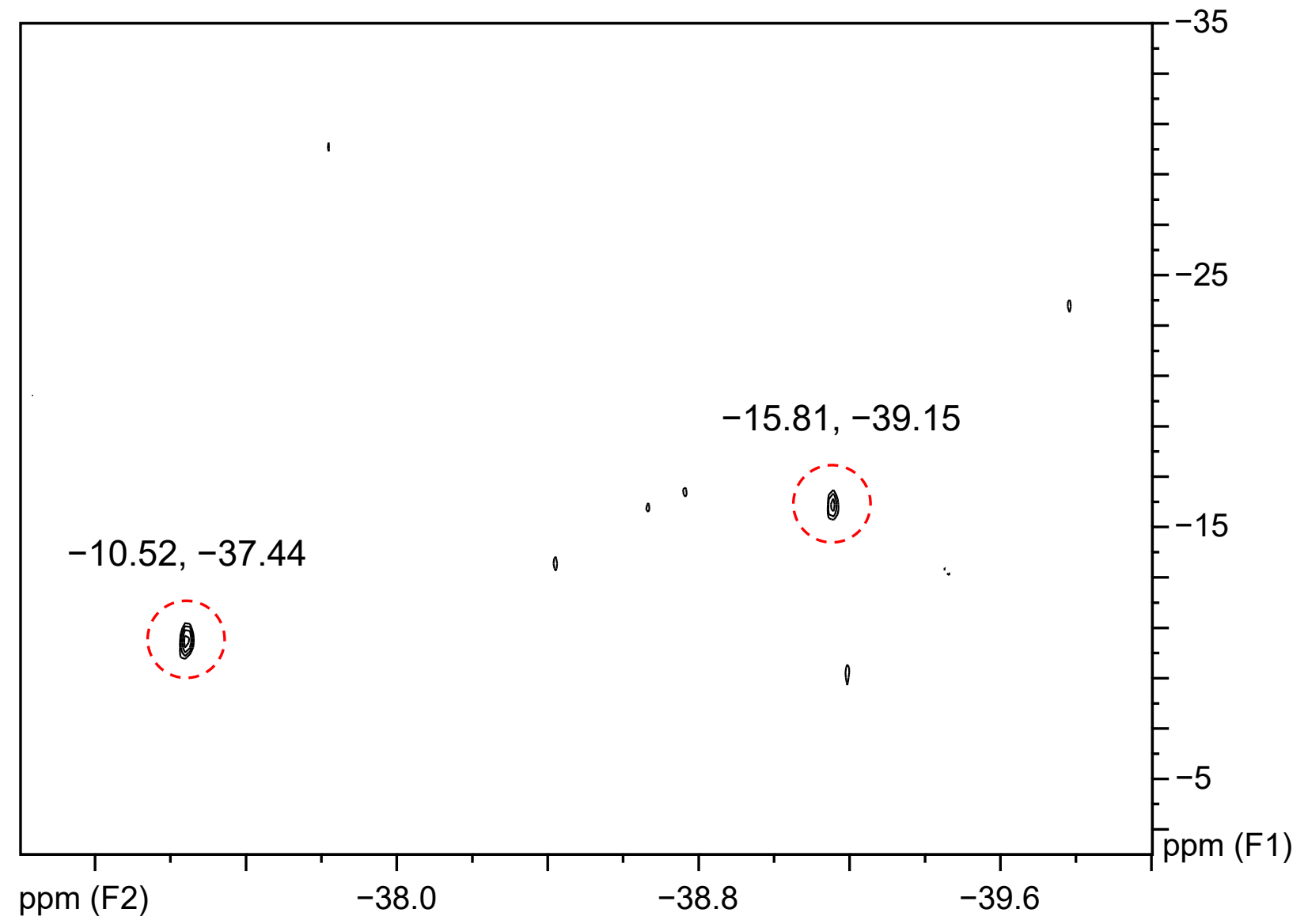

Figure S2. ${ }^{29} \mathrm{Si}$ NMR COSY spectrum at $5{ }^{\circ} \mathrm{C}$ of the solution represented in Figure $1 \mathrm{a}(1.5 \mathrm{~mol}$ $\mathrm{kg}^{-1}{ }^{29} \mathrm{SiO}_{2}, 1.5 \mathrm{~mol} \mathrm{~kg}{ }^{-1} \mathrm{KOH}$ and $1.2 \mathrm{~mol} \mathrm{~kg}^{-1}$ mannitol), ${ }^{2}$ expanded to show cross peaks for the $\underline{\mathrm{Q}}^{n} \underline{\mathrm{P}}^{1}(=\mathrm{L})_{2}$ cluster of resonances at $-38 \mathrm{ppm}$. Correlations were evident with signals at -10.52 and $-15.81 \mathrm{ppm}$, which would be consistent with $n=2$ (or possibly 3 ). 


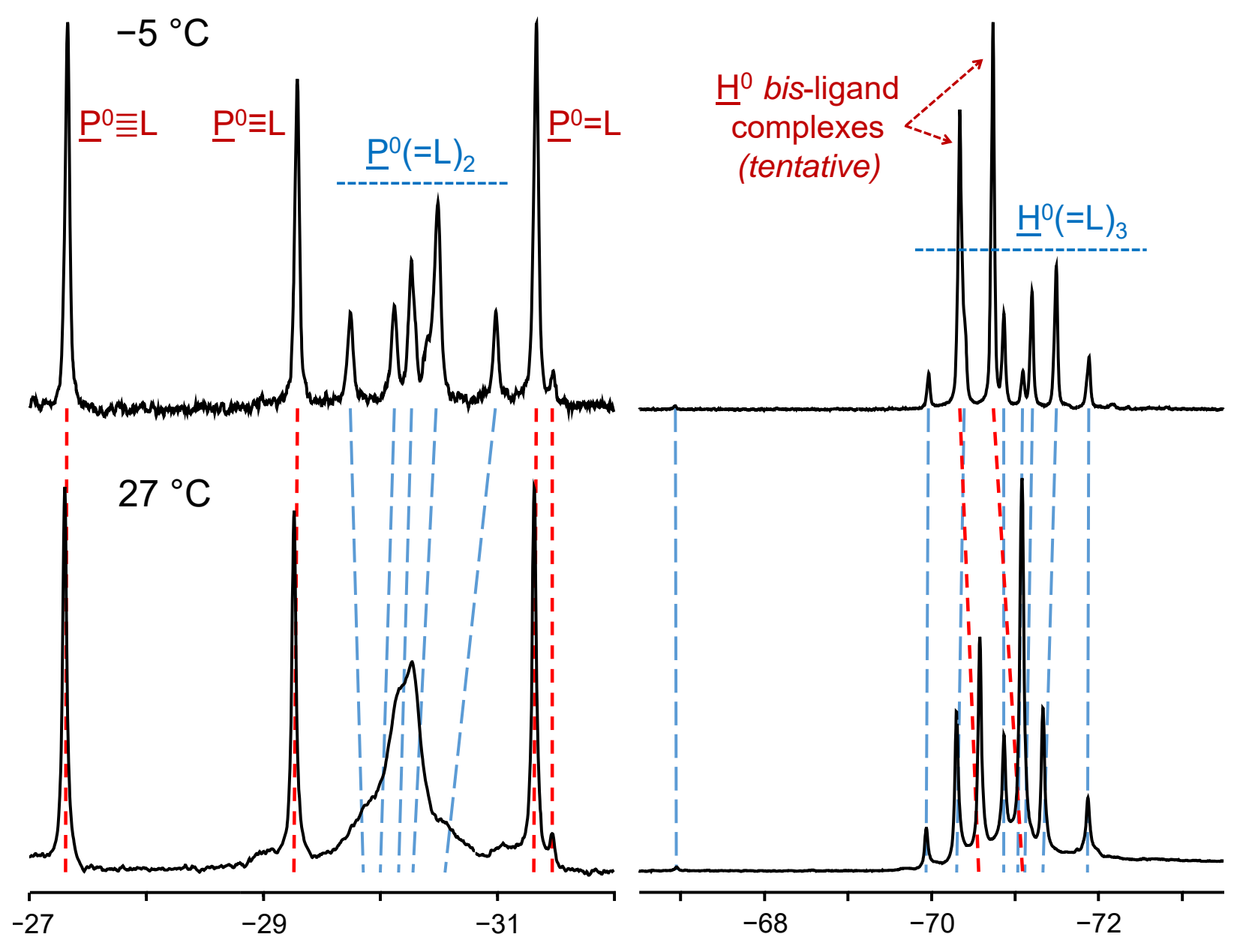

Figure S3. ${ }^{29} \mathrm{Si}\left\{{ }^{1} \mathrm{H}\right\}$ NMR spectra at $-5{ }^{\circ} \mathrm{C}$ and $27{ }^{\circ} \mathrm{C}$ of the silicate-glucoheptonate solution represented in Figure 5. ${ }^{2}$ 

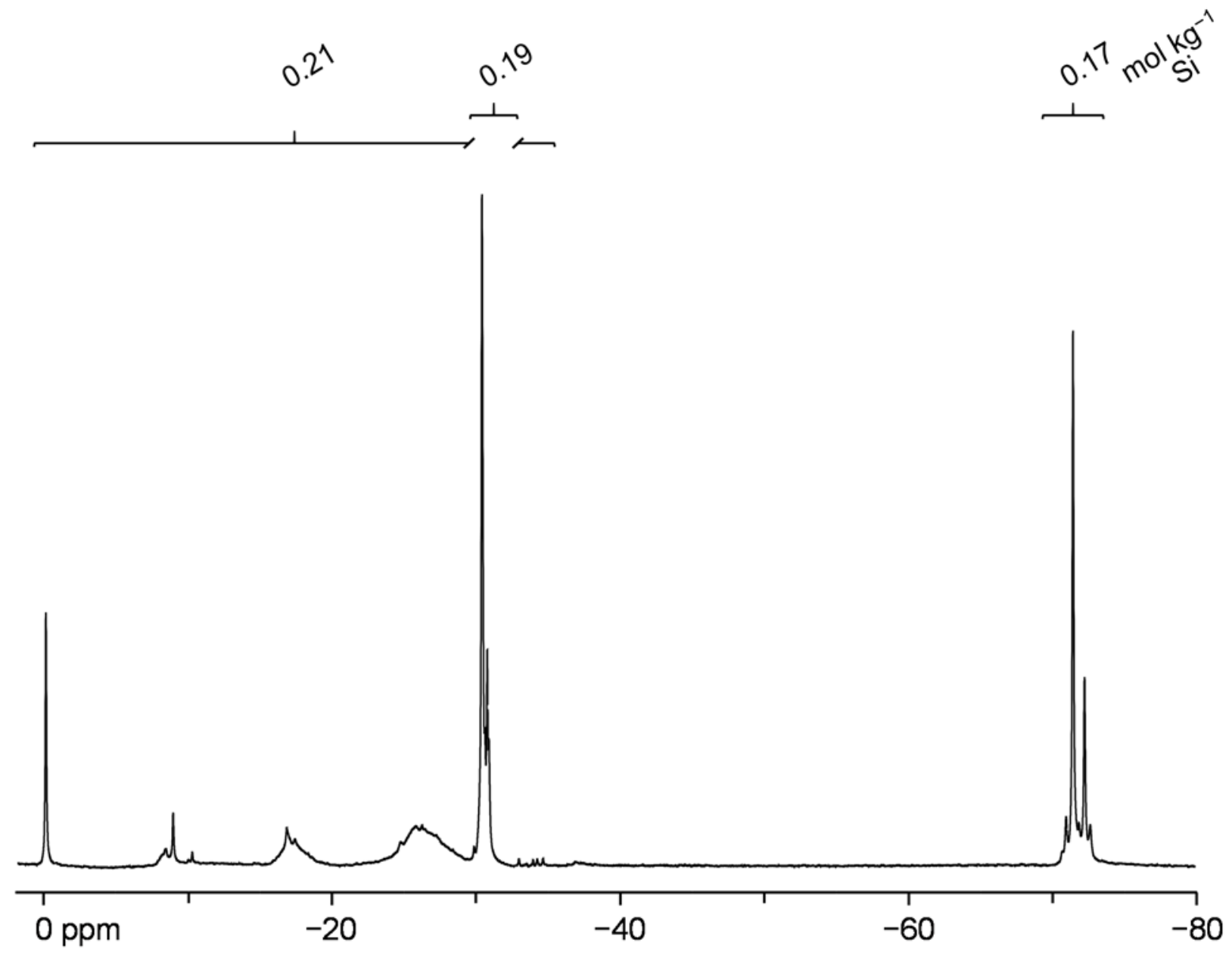

Figure S4. ${ }^{29} \mathrm{Si} \mathrm{NMR}$ spectrum $\left({ }^{1} \mathrm{H}\right.$-coupled $)$ at $5{ }^{\circ} \mathrm{C}$ of a solution containing $0.57 \mathrm{~mol} \mathrm{~kg}{ }^{-1}{ }^{29} \mathrm{SiO}_{2}$, $0.57 \mathrm{~mol} \mathrm{~kg}^{-1} \mathrm{NaOH}$ and $0.58 \mathrm{~mol} \mathrm{~kg}^{-1}$ iditol. If the dominant 5- and 6-coordinate Si species were respectively $\underline{\mathrm{P}}^{0}(=\mathrm{L})_{2}$ and $\underline{\mathrm{H}}^{0}(=\mathrm{L})_{3}$, as in most other silicate-polyol systems, the concentration of complexed ligand would equal $0.89 \mathrm{~mol} \mathrm{~kg}^{-1}$, that is, $150 \%$ of the total ligand in solution. If instead they were $\underline{\mathrm{P}}^{0}$ mono-ligand and $\underline{\mathrm{H}}^{0}$ bis-ligand species, complexed iditol would represent $93 \%$ of the total solution content. 
a)

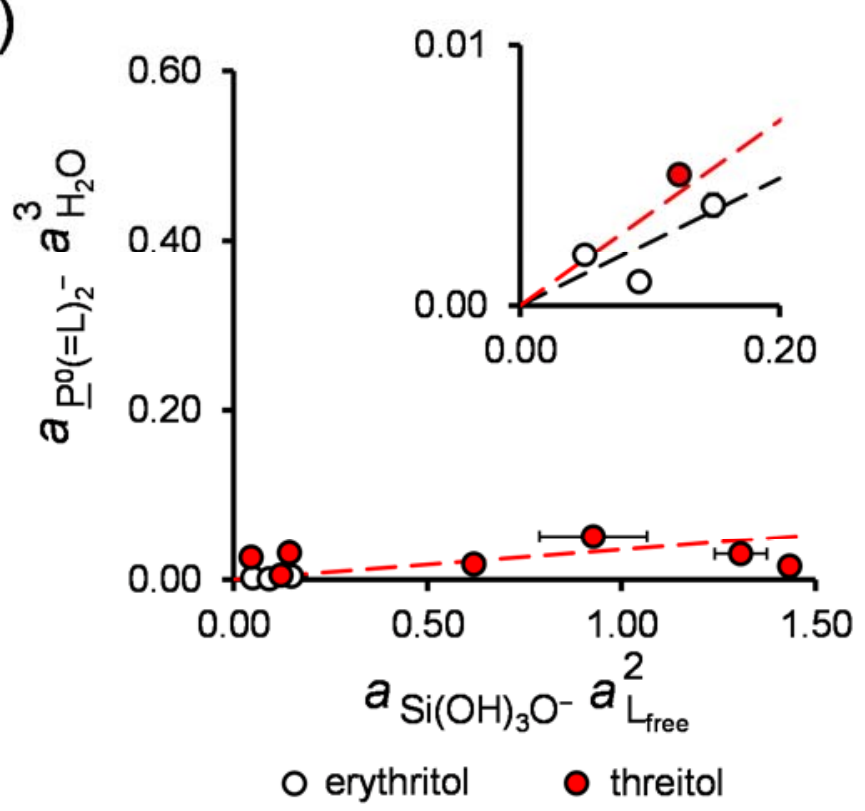

b)

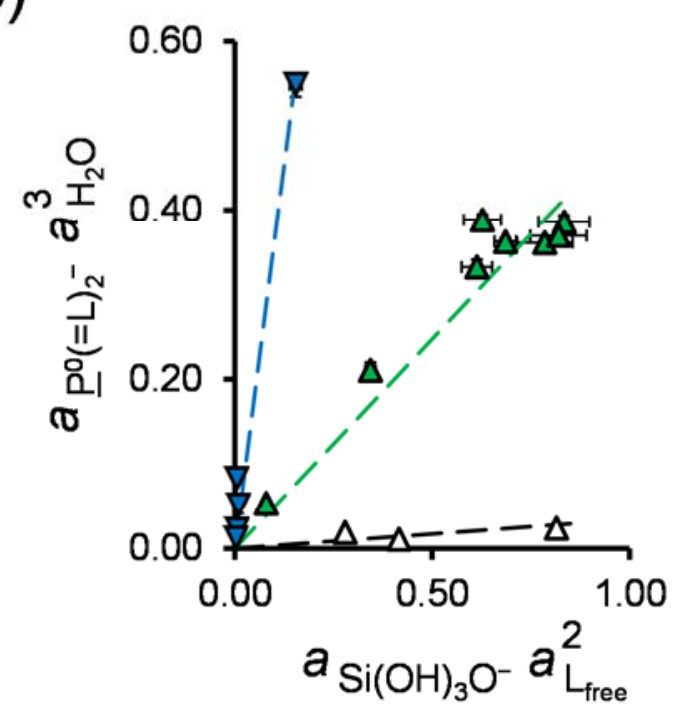

$\Delta$ adonitol $\quad \Delta$ arabitol $\quad \nabla$ xylitol

Figure S5. Graphical determination of $\beta_{\mathrm{P}^{0}(=\mathrm{L})_{2}}$ at $5{ }^{\circ} \mathrm{C}$ for: a) erythritol and threitol; and b) adonitol, arabitol and xylitol.

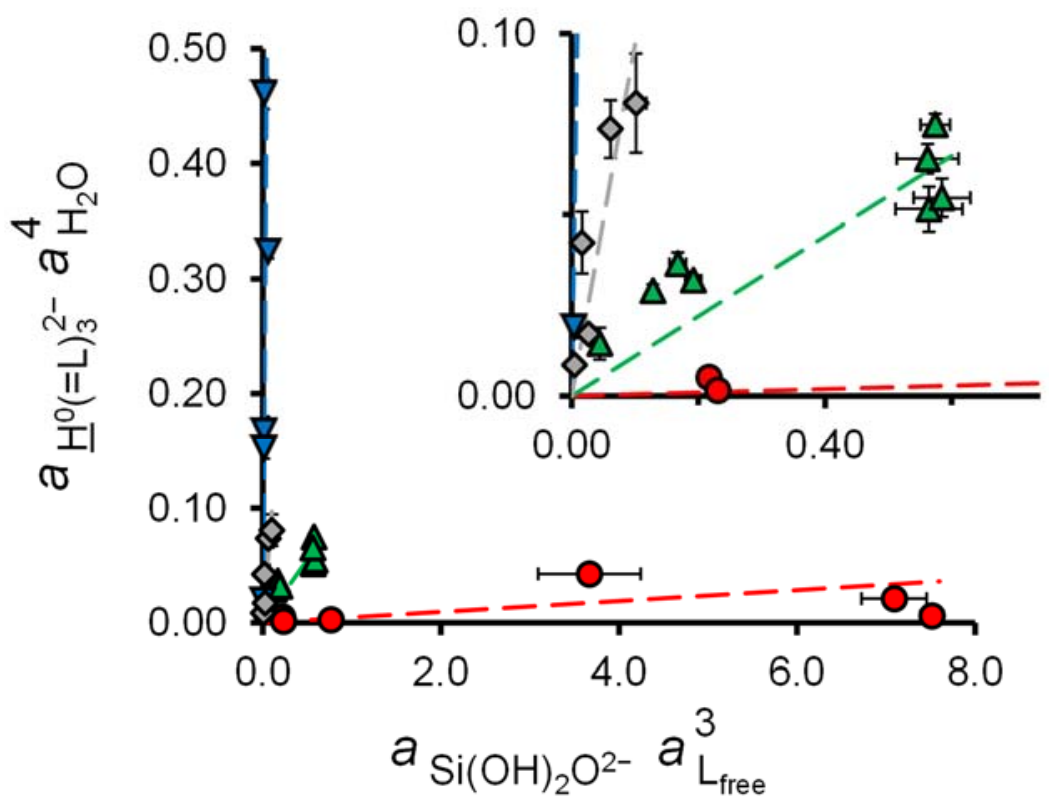

○ threitol $\Delta$ arabitol $\boldsymbol{\nabla}$ xylitol $\diamond$ mannitol

Figure S6. Graphical determination of $\beta_{\mathrm{H}^{0}(=\mathrm{L})_{3}}$ at $5{ }^{\circ} \mathrm{C}$ for threitol, arabitol, xylitol and mannitol. 


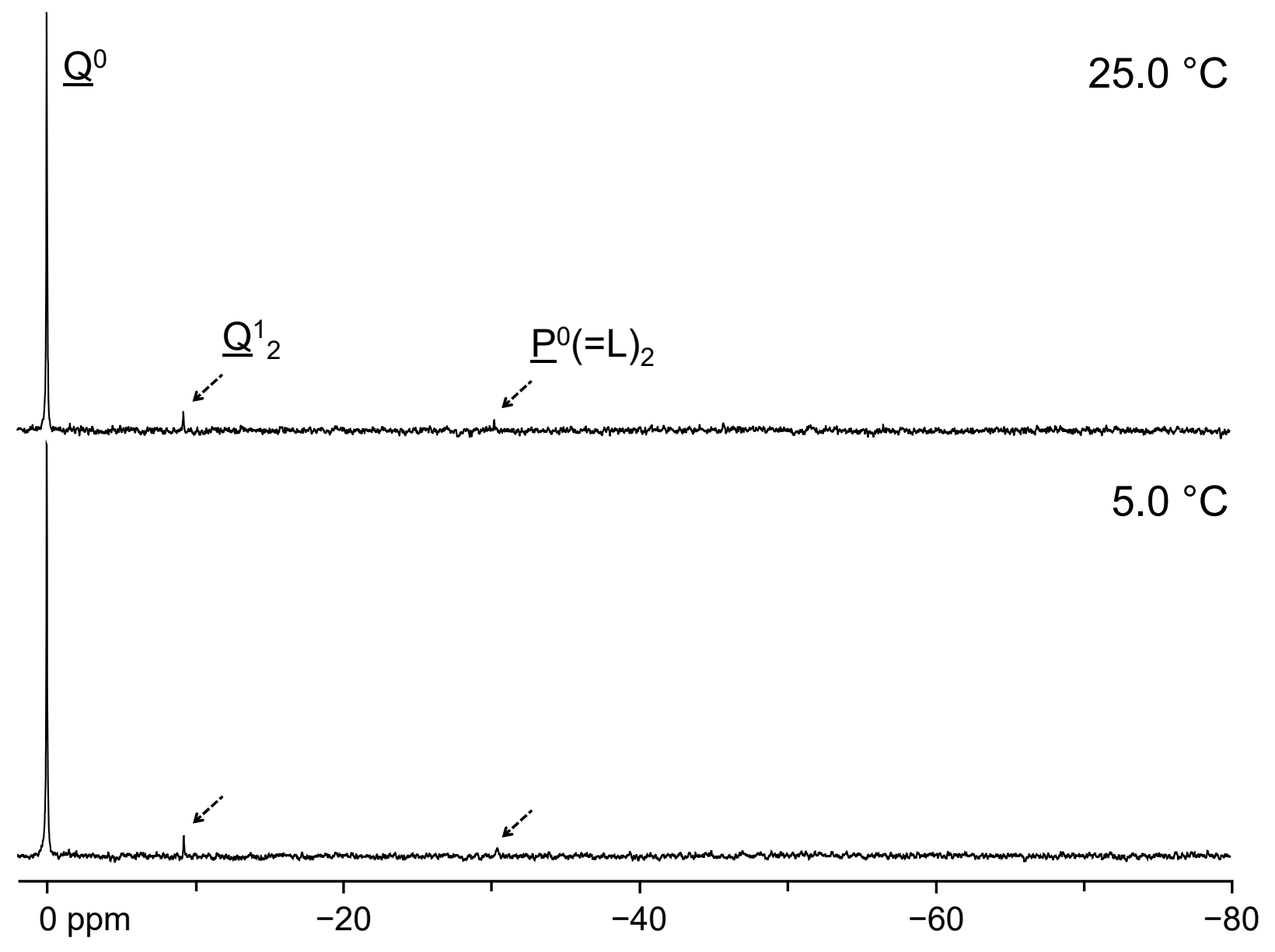

Figure S7. ${ }^{29} \mathrm{Si}\left\{{ }^{1} \mathrm{H}\right\} \mathrm{NMR}$ spectra at $5{ }^{\circ} \mathrm{C}$ and $25^{\circ} \mathrm{C}$ of a pH 7.05 (measured at $5{ }^{\circ} \mathrm{C}$ ) solution containing $1.71 \mathrm{mmol} \mathrm{kg}^{-1}{ }^{29} \mathrm{SiO}_{2}$ and $2.97 \mathrm{~mol} \mathrm{~kg}^{-1}$ xylitol. They were each recorded using $2560 \pi / 2$ pulses with a recycle period of $93 \mathrm{~s}$ (thus, 5.5 days total acquisition). The fraction of dissolved Si existing in the form of $\underline{\mathrm{P}}^{0}(=\mathrm{L})_{2}$, corresponding to at least two signals at $-30.5 \mathrm{ppm}$, was $3.0 \pm 0.5 \%$ over this temperature range. 
The total pool of monomeric silicate may be determined by combining equations (1) to (4b) from ref. 2 :

$$
\begin{aligned}
& a_{\mathrm{Si}_{\mathrm{T}}}=a_{\mathrm{Si}(\mathrm{OH})_{4}}+a_{\mathrm{Si}(\mathrm{OH})_{3} \mathrm{O}^{-}}+a_{\mathrm{Si}(\mathrm{OH})_{2} \mathrm{O}_{2}{ }^{2-}}+a_{\mathrm{P}^{0}(=\mathrm{L})_{2}}+a_{\mathrm{H}^{0}(=\mathrm{L})_{3}} \\
& =\frac{a_{\mathrm{P}^{0}(=\mathrm{L})_{2}} a_{\mathrm{H}^{+}} a_{\mathrm{H}_{2} \mathrm{O}^{3}}}{K_{\mathrm{a} 1} \beta_{\mathrm{P}^{0}(=\mathrm{L})_{2}} a_{\mathrm{L}_{\text {free }}}{ }^{2}}+\frac{a_{\mathrm{P}^{0}(=\mathrm{L})_{2}} a_{\mathrm{H}_{2} \mathrm{O}^{3}}}{\beta_{\mathrm{P} \mathrm{O}_{(=\mathrm{L})_{2}}} a_{\mathrm{L}_{\text {free }}}{ }^{2}}+\frac{K_{\mathrm{a} 2} a_{\mathrm{P}^{0}(=\mathrm{L})_{2}} a_{\mathrm{H}_{2} \mathrm{O}^{3}}}{\beta_{\mathrm{P}^{0}(=\mathrm{L})_{2}} a_{\mathrm{L}_{\mathrm{free}}}{ }^{2} a_{\mathrm{H}^{+}}}+a_{\mathrm{P}^{0}(=\mathrm{L})_{2}}+\frac{K_{\mathrm{a} 2} \beta_{\mathrm{H}^{0}(=\mathrm{L})_{3}} a_{\mathrm{P}^{0}(=\mathrm{L})_{2}} a_{\mathrm{L}_{\mathrm{free}}}}{\beta_{\mathrm{P}^{0}(=\mathrm{L})_{2}} a_{\mathrm{H}^{+}} a_{\mathrm{H}_{2} \mathrm{O}}} \\
& =a_{\mathrm{P}^{0}(=\mathrm{L})_{2}}\left(\frac{a_{\mathrm{H}^{+}} a_{\mathrm{H}_{2} \mathrm{O}^{3}}}{K_{\mathrm{a} 1} \beta_{\mathrm{P}^{0}(=\mathrm{L})_{2}} a_{\mathrm{L}_{\text {free }}}^{2}}+\frac{a_{\mathrm{H}_{2} \mathrm{O}^{3}}}{\beta_{\mathrm{P}^{0}(=\mathrm{L})_{2}} a_{\mathrm{L}_{\text {free }}}^{2}}+\frac{K_{\mathrm{a} 2} a_{\mathrm{H}_{2} \mathrm{O}^{3}}}{\beta_{\mathrm{P}^{0}(=\mathrm{L})_{2}} a_{\mathrm{L}_{\text {free }}} a_{\mathrm{H}^{+}}}+1+\frac{K_{\mathrm{a} 2} \beta_{\mathrm{H}^{0}(=\mathrm{L})_{3}} a_{\mathrm{L}_{\text {free }}}}{\beta_{\mathrm{P}^{0}(=\mathrm{L})_{2}} a_{\mathrm{H}^{+}} a_{\mathrm{H}_{2} \mathrm{O}}}\right)
\end{aligned}
$$

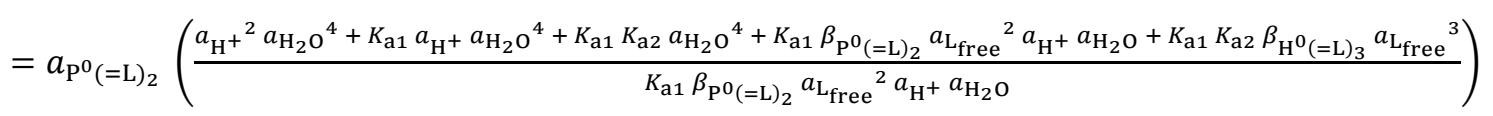

Equations (3b) and ( $4 b)$ can therefore be rearranged to give:

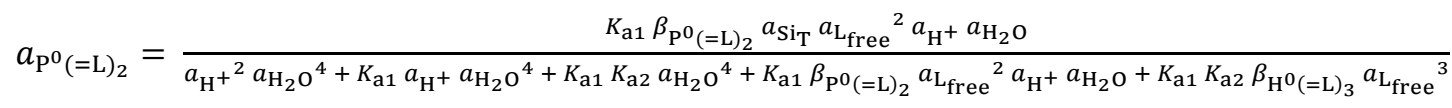

$$
\begin{aligned}
& a_{\mathrm{H}^{0}(=\mathrm{L})_{3}}=\frac{K_{\mathrm{a} 2} \beta_{\mathrm{H}^{0}(=\mathrm{L})_{3}} a_{\mathrm{P}^{0}(=\mathrm{L})_{2}} a_{\mathrm{L}_{\text {free }}}}{\beta_{\mathrm{P}^{0}(=\mathrm{L})_{2}} a_{\mathrm{L}_{\mathrm{free}}}{ }^{2} a_{\mathrm{H}^{+}} a_{\mathrm{H}_{2} \mathrm{O}}}
\end{aligned}
$$

Scheme S1. Calculation of activity values for $\underline{\mathrm{P}}^{0}(=\mathrm{L})_{2}$ and $\underline{\mathrm{H}}^{0}(=\mathrm{L})_{3}$.

\section{- REFERENCES}

1. Jesus, A. J. L.; Tome, L. I. N.; Rosado, M. T. S.; Leitao, M. L. P.; Redinha, J. S. Conformational study of erythritol and threitol in the gas state by density functional theory calculations. Carbohydr. Res. 2005, 340, 283-291.

2. Vis, B. M.; Wen, J.; Mellerup, S. K.; Merchant, R. D.; Mawhinney, R. C.; Kinrade, S. D. Silicon forms a rich diversity of aliphatic polyol complexes in aqueous solution. J. Am. Chem. Soc. 2020. DOI: 10.1021/jacs.9b10701 